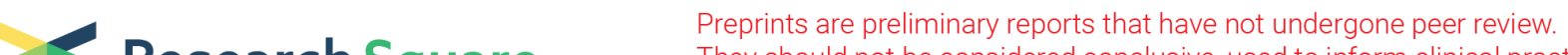 Research Square They should not be considered conclusive, used to inform clinical practice, or referenced by the media as validated information.
}

\section{Upregulation of long intergenic noncoding RNA LINC00092 indicates favorable survival in lung adenocarcinoma via tumor growth inhibition}

\section{Mingyan Jiang}

Xiangtan Central Hospital

Ke Wang ( $\square$ majikcan@126.com )

Guangzhou Medical University https://orcid.org/0000-0002-2575-1826

\section{Ziwen Zhao}

Guangzhou First People's Hospital

\section{Yujun Li}

Guangzhou First People's Hospital

\section{Hua He}

Guangzhou First People's Hospital

Shuquan Wei

Guangzhou First People's Hospital

Quhai Chen

Guangzhou First People's Hospital

Mindong Chen

Guangzhou First People's Hospital

Jianjiang Xie

Guangzhou First People's Hospital

Zhuxiang Zhao

Guangzhou First People's Hospital

\section{Research article}

Keywords: LINC00092, survival, lung adenocarcinoma, TCGA

Posted Date: November 26th, 2019

DOI: https://doi.org/10.21203/rs.2.17845/v1

License: (c) (i) This work is licensed under a Creative Commons Attribution 4.0 International License. Read Full License 


\section{Abstract}

Background Long intergenic noncoding RNA 00092 (LINC00092) is a recently identified novel RNA that acts on cancer-associated fibroblasts (CAFs) to drive the progression of ovarian cancer. Because CAFs also play a vital role in lung cancer, we hypothesized that LINC00092 is associated with lung cancer.Methods The expression level of LINC00092 was examined in 93 cases of non-small cell lung cancer (NSCLC) tumor tissues and adjacent normal lung tissues, and its clinical effect on prognosis was evaluated using the Kaplan-Meier method, log-rank test and Cox regression analysis based on The Cancer Genome Atlas (TCGA) data. The effect of LINC00092 on tumor growth was further assessed in vitro .Results LINC00092 was significantly downregulated in 76.3\% (71/93) of lung cancer tissues compared to that in their normal counterparts $(P<0.001$ ), and high expression of LINC00092 led to a better prognosis with increased survival time (1632 days vs. 1171 days; $P=0.087)$ and decreased mortality (hazard rate, $\mathrm{HR}=0.73,95 \% \mathrm{Cl}=0.51-1.05$ ) than low expression in lung adenocarcinoma (LUAD) but not in lung squamous cell carcinoma (LSCC) patients. Additionally, upregulation of LINC00092 inhibited LUAD cell proliferation and tumorigenic ability in vitro .Conclusions LINC00092 is an indicator of favorable LUAD prognosis. Targeted molecular therapy directed at LINC00092 upregulation may be a valuable strategy to fight LUAD.

\section{Background}

Lung cancer has ranked as the leading cause of cancer-related death for several years. According to data from the American Cancer Society, 158,080 lung cancer deaths were estimated in America in 2016, accounting for $26.5 \%$ of all cancer deaths (Siegel, Miller et al., 2016). Data from the National Central Cancer Registry of China also showed that the lung cancer mortality was $610.2 / 10^{5}$ in 2015, ranking first among all cancer deaths (Chen, Zheng et al., 2016). The 5-year survival rate of lung cancer patients is still low, with values lower than $20 \%$. However, advancements in treatment strategies for lung cancer have improved in lung cancer survival rates ranging from $12 \%$ in 1975 to $1977,13 \%$ in $1987-1989$ to $18 \%$ in $2004-2010$ (Siegel, Miller et al., 2015). The effect is small but significant. This increase in the last two decades is mainly due to targeted molecular therapy, such as tyrosine kinase inhibitors targeting the epidermal growth factor receptor used in the treatment of lung cancer, which has resulted in considerable improvements in overall survival, indicating promising prospects in the fight against lung cancer (Chen, Kronenberger et al., 2012; Chee, Robinson et al., 2017). Nevertheless, the downside is that patient responses to targeted anticancer agents are discrepant and variable, which may be a major reason limiting improvement in lung cancer survival. Thus, testing for predictive biomarkers for targeted therapy is becoming an important part of the diagnostic workup. We still need to exert great efforts to identify effective prognostic biomarkers.

Long intergenic noncoding RNAs (lincRNAs) are defined as intergenic non-protein coding transcripts longer than 200 nucleotides. Thus far, many lincRNAs have been identified as possible oncogenes or tumor suppressors that drive or inhibit tumorigenesis and cancer progression. Further, some lincRNAs, such as LINC00673 (Ma, Wu et al., 2017), LINC00511 (Sun, Li et al., 2016), LINC00672 (Li, Li et al., 2017), HULC (Panzitt, Tschernatsch et al., 2007) and CASC15 (Russell, Penikis et al., 2015), have been suggested to be possible diagnostic and prognostic biomarkers or therapeutic targets owing to their significant correlation with clinical features in cancer. However, there is still little knowledge on the role of lincRNAs in lung cancer. 
LINC00092 (NR_024129.1) is a newly identified lincRNA that is upregulated in ovarian cancer and correlated with poor prognosis in patients (Zhao, Ji et al., 2017). Mechanistic analyses have shown that LINC00092 can induce cancer-associated fibroblasts (CAFs) to promote metastasis of cancer cells (Zhao, Ji et al., 2017). CAFs also play a definite role in lung cancer development (Micke and Ostman, 2004). We thus hypothesized that LINC00092 is associated with the progression of lung cancer. In the current study, we evaluated the expression levels of LINC00092 in 132 pairs of non-small cell lung cancer (NSCLC) tissues and adjacent normal lung tissues to assess its clinical significance. Data from The Cancer Genome Atlas (TCGA) database and functional analyses were used to respectively validate the association and reveal the underlying mechanism.

\section{Methods}

\section{Tissue sample collection}

NSCLC tissues and their corresponding adjacent normal tissues were collected from 98 NSCLC patients who underwent surgery at the Guangzhou First People's Hospital from January 2012 to July 2016. All tissue samples were immediately frozen in liquid nitrogen after being washed with phosphate-buffered saline (PBS). Each patient provided an informed written consent form. All patients were followed up for vital status until the end of November 2016, and 132 cases were successfully followed up with complete survival data. Data on clinicopathological parameters, including histopathology, TNM status and American Joint Committee on Cancer (AJCC) stage, as well as demographic characteristics, including age, sex and smoking status, were extracted from medical records. The study was approved by the institutional review board at the Guangzhou First People's Hospital. All methods were performed in accordance with relevant guidelines and regulations.

\section{RNA extraction and RT-PCR}

Total RNA was extracted from all tissue samples using TRIzol reagent (Invitrogen, Carlsbad, USA) according to the manufacturer's protocol. The expression level of LINC00092 and that of the internal reference $\beta$-actin were detected using routine SYBR green quantitative reverse real-time PCR (qRT-PCR) on ABI 7900 system (ThermoFisher, USA). The primer sequences for LINC00092 and $\beta$-actin were synthesized by Invitrogen. The sequences of primers were as suggested (Yang, Yang et al., 2014; Zhao, Ji et al., 2017).

\section{TCGA NSCLC datasets and the LINC00092 coexpression network}

Available data on LINC00092 expression were derived from 979 NSCLC patients (i.e., 495 LUAD and 484 LSCC patients) with definite survival follow-up data in TCGA portal (https://portal.gdc.cancer.gov/). Analysis of differential gene expression between NSCLC tissues and their normal counterparts were performed in all of the above samples. The LINC00092 coexpression network was constructed based only on the LUAD data. WGCNA in

R software was used to produce expression modules (Langfelder and Horvath, 2008). Cytoscape was employed to construct the network diagram (Shannon, Markiel et al., 2003).

\section{LINC00092 overexpression vector construction and cell transfection}

The whole transcript of LINC00092 was cloned into the overexpression vector pcDNA3.1. The empty vector pcDNA3.1 was used as a control. The vectors were transiently transfected into two LUAD cell lines, A549 and PC- 
9. The cells were placed in a $\mathrm{CO}_{2}$-containing incubator (SANYO Electric Co., Ltd., Japan) with constant $90 \%$ humidity and $5 \% \mathrm{CO}_{2}$.

Tumor growth assay in vitro

Cell proliferation was tested using the Cell Counting Kit-8 (CCK8) assay according to the manufacturer's protocols. LUAD cells were seeded in a 96-well plate, and cell viability was measured after 24, 48, 72 and 96 h. The clonogenic ability of LUAD cells was assessed via plate colony assays. Cells were plated in a 6-well plate (100 cells/well) and cultured for two weeks. Then, the visible colonies were counted after staining. The clonogenic ability of LUAD cells was also analyzed using soft agar assays. Cell suspensions were mixed with $0.35 \%$ soft agar in medium containing $10 \%$ FBS and layered in triplicate on $0.75 \%$ solidified agar in the same growth medium ( $3 \times$ $10^{3}$ cells/well). After three weeks of culture, colonies were stained, photographed and counted.

\section{Transwell migration and invasion assays}

Cell migration and invasion were respectively tested using Corning Transwell insert chambers and BD BioCoat Matrigel Invasion Chambers in accordance with the manufacturer's protocols. Generally, $2 \times 10^{4}$ or $2 \times 10^{5}$ cells in serum-free RPMI 1640 medium were cultured in the upper chamber for migration and invasion, respectively, cell medium with $10 \%$ FBS was added to the lower compartment. After $24 \mathrm{~h}$ (for migration) or $48 \mathrm{~h}$ (for invasion), cells in the upper chamber were carefully scraped off using a cotton swab, and cells in the lower surfaces of the membrane were counted.

\section{Statistical analysis}

Differences in gene expression between NSCLC tumor tissues and their normal counterparts were tested by paired $\mathrm{t}$ tests. Comparisons between groups were performed via Student's $\mathrm{t}$ test or one-way ANOVA. Survival curves were constructed using the Kaplan-Meier method. The effect of LINC00092 on survival was evaluated using the logrank test, while the strength of association between LINC00092 expression and survival was calculated by Cox models adjusted for age, sex, smoking status, histological type and clinical stage. All tests were two-sided and performed using Stata 12.0 software, and a P value less than 0.05 was considered statistically significant.

\section{Results}

\section{LINC00092 is downregulated in NSCLC tissues}

A total of 98 samples were collected to detect LINC00092 expression, and 93 cases among them were finally included in the study using the detection of expression levels of LINC00092 in both cancerous and normal tissues. Unlike its upregulation in ovarian cancer (Zhao, Ji et al., 2017), we found that LINC00092 was significantly downregulated in $76.3 \%$ (71/93) of lung cancer tissues compared to that in their normal counterparts $(P<0.001$; Fig. 1a). The expression levels of LINC00092 were significantly higher in lung cancer tissues than in adjacent normal lung tissues (mean \pm standard deviation: $0.208 \pm 0.327$ vs. $0.435 \pm 0.255 ; P<0.001$; Fig. 1 b).

\section{LINC00092 may be correlated with metastasis in the total cohort}


We assessed the correlation between LINC00092 expression levels in cancer tissues and clinicopathological parameters. As shown in Table1, the results revealed that LINC00092 expression levels were likely to be correlated with the presence or absence of metastasis $(M)$, as a lower level was observed in patients with positive metastasis $(0.144 \pm 0.272)$ than in patients with negative metastasis $(0.258 \pm 0.359)$. The difference showed a clear tendency to being significant $(P=0.096)$. However, no significant or slightly significant $(P<0.1)$ associations were observed between LINC00092 expression and primary tumor dissociation ( $T ; P=0.357$ ), extent of lymph node involvement $(\mathrm{N} ; \mathrm{P}=0.191)$ or clinical stage $(\mathrm{P}=0.311)$. 
Table 1

Effect of LINC00092 on survival in NSCLC.

\begin{tabular}{|c|c|c|c|c|c|c|c|c|c|}
\hline \multirow[t]{2}{*}{ Parameter } & \multicolumn{3}{|c|}{$\begin{array}{l}\text { Low LINC00092 } \\
\text { expression }\end{array}$} & \multicolumn{3}{|c|}{$\begin{array}{l}\text { High LINC00092 } \\
\text { expression }\end{array}$} & \multirow{2}{*}{$\begin{array}{l}\text { Log- } \\
\text { rank } \\
\mathrm{P} \\
\text { value }\end{array}$} & \multirow{2}{*}{$\begin{array}{l}\begin{array}{l}\text { HR } \\
(95 \% \mathrm{Cl})^{a}\end{array} \\
\text { High vs. } \\
\text { Low }\end{array}$} & \multirow{2}{*}{$\begin{array}{l}P \\
\text { value } \\
\text { a }\end{array}$} \\
\hline & $\mathrm{n}$ & Death & $\begin{array}{l}\text { MST } \\
\text { (months) }\end{array}$ & $\begin{array}{l}n \\
(\%)\end{array}$ & Death & $\begin{array}{l}\text { MST } \\
\text { (months) }\end{array}$ & & & \\
\hline Total & 68 & 53 & 15 & 64 & 38 & 19 & 0.145 & $\begin{array}{l}0.67(0.43- \\
1.03)\end{array}$ & 0.069 \\
\hline \multicolumn{10}{|l|}{ Histological type } \\
\hline Adenocarcinoma & 33 & 27 & 12 & 32 & 17 & 27 & 0.025 & $\begin{array}{l}0.56(0.30- \\
1.05)\end{array}$ & 0.072 \\
\hline $\begin{array}{l}\text { Squamous cell } \\
\text { carcinoma }\end{array}$ & 18 & 14 & 20 & 22 & 14 & 18 & 0.846 & $\begin{array}{l}0.53(0.22- \\
1.23)\end{array}$ & 0.138 \\
\hline Other ${ }^{b}$ & 17 & 12 & 16 & 10 & 7 & 14 & 0.669 & $\begin{array}{l}1.80(0.31- \\
6.32)\end{array}$ & 0.358 \\
\hline \multicolumn{10}{|l|}{ Tumor size } \\
\hline$\leq 5 \mathrm{~cm}$ & 31 & 21 & 19 & 22 & 10 & 27 & 0.179 & $\begin{array}{l}0.65(0.29- \\
1.45)\end{array}$ & 0.291 \\
\hline$>5 \mathrm{~cm}$ & 36 & 31 & 10 & 39 & 26 & 19 & 0.241 & $\begin{array}{l}0.65(0.36- \\
1.17)\end{array}$ & 0.149 \\
\hline \multicolumn{10}{|l|}{$\begin{array}{l}\text { Nodal } \\
\text { metastasis }\end{array}$} \\
\hline Negative & 23 & 13 & 35 & 16 & 2 & - & 0.015 & $\begin{array}{l}0.17(0.03- \\
0.86)\end{array}$ & 0.031 \\
\hline Positive & 44 & 39 & 10 & 47 & 35 & 16 & 0.200 & $\begin{array}{l}0.89(0.55- \\
1.44)\end{array}$ & 0.631 \\
\hline \multicolumn{10}{|l|}{$\begin{array}{l}\text { Distal } \\
\text { metastasis }\end{array}$} \\
\hline Negative & 34 & 21 & 28 & 32 & 14 & 27 & 0.910 & $\begin{array}{l}0.90(0.45- \\
1.83)\end{array}$ & 0.779 \\
\hline Positive & 33 & 32 & 9 & 30 & 24 & 10 & 0.092 & $\begin{array}{l}0.63(0.35- \\
1.14)\end{array}$ & 0.128 \\
\hline \multicolumn{10}{|l|}{ Clinical stage } \\
\hline I/II & 19 & 9 & 38 & 17 & 5 & - & 0.728 & $\begin{array}{l}1.06(0.32- \\
3.58)\end{array}$ & 0.921 \\
\hline
\end{tabular}

Abbreviation: MST, median survival time.

${ }^{a}$ Cox regression analysis was adjusted for age, sex, smoking status, histological type and clinical stage.

${ }^{b}$ Mixed or unknown. 


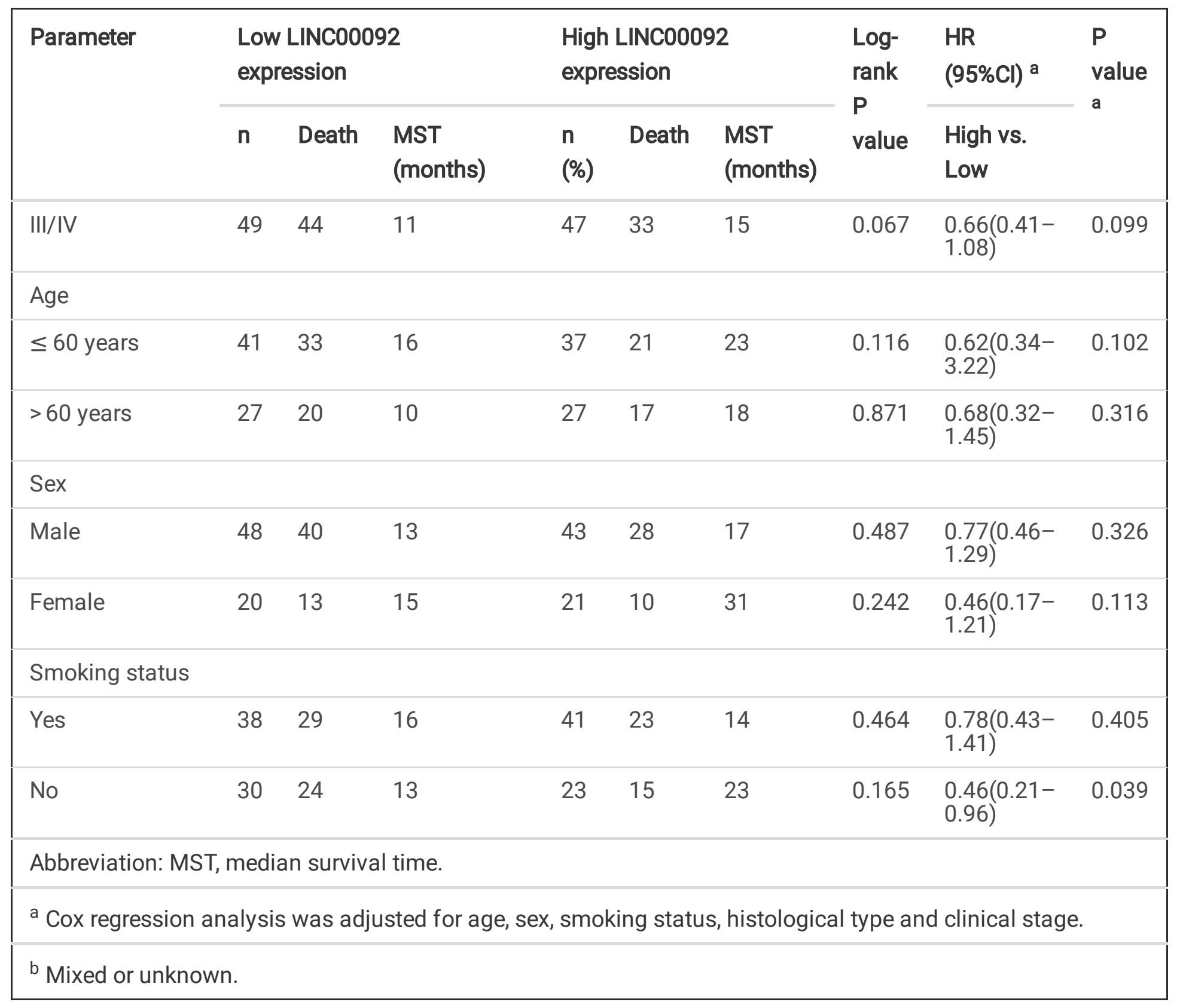

\section{High expression of LINC00092 mediates favorable survival in lung adenocarcinoma (LUAD)}

We tested the effect of LINC00092 expression on NSCLC patient survival based on TCGA data

(https://cancergenome.nih.gov/). The patients were divided into two groups, a high LINC00092 expression group (<median value) and a low LINC00092 expression group (> median value). As shown in Fig. 1c-d, both the log-rank test and Cox models indicated that high LINC00092 expression confers favorable survival in LUAD patients (high vs. low: 1622 days vs. 1171 days, log-rank $\mathrm{P}=0.087$; hazard rate, $\mathrm{HR}=0.73,95 \% \mathrm{Cl}=0.51-1.05$ ) but not in LSCC patients (high vs. low: 1656 days vs. 1423 days, log-rank $\mathrm{P}=0.450 ; \mathrm{HR}=0.88,95 \% \mathrm{Cl}=0.64-1.22$ ). The favorable survival effect of LINC00092 in LUAD displays a substantial trend toward significance.

\section{Upregulated LINC00092 inhibits LUAD cell growth}


The whole LINC00092 transcript was cloned into the pcDNA3.1 vector and transfected into two LUAD cell lines, A549 and PC-9. Upregulation of LINC00092 was confirmed in both cell lines transfected with pcDNA3.1-

LINC00092 in comparison to that in control cells transfected with empty pcDNA3.1 vectors (Figure S1). The effect of LINC00092 on cell growth was tested by Cell Counting Kit-8 (CCK8), plate colony and soft agar assays. As shown in Fig. 2a, upregulation of LINC00092 apparently slowed the proliferation rate of LUAD cells. Further, cells overexpressing LINC00092 showed weaker growth ability in plate colony and soft agar assays (Fig. 2a, b).

\section{Upregulation of LINC00092 does not impair migration and invasion in LUAD cells}

A previous study reported a beneficial role of LINC00092 on ovarian cancer cell migration and invasion (Zhao, Ji et al., 2017); thus, using Transwell assays, we investigated whether LINC00092 affects the migration and invasion of LUAD cells. The assay revealed that upregulation of LINC00092 did not significantly affect either migratory or invasive ability of LUAD cells (Fig. 3).

\section{Gene coexpression network with regard to LINC00092}

To evaluate possible coding genes related to LINC00092, we constructed a coexpression network based on TCGA data using Weighted Correlation Network analysis (WGCNA) in R. The analysis revealed that LINC00092 expression displays significant, positive and substantial correlations with 11 coding genes (Fig. 4a), including glutathione S-transferase mu 5 (GSTM5: $\left.r=0.607, P=1.23 \times 10^{-58}\right)$, EF-hand and coiled-coil domain-containing 1 (EFCC1: $\left.r=0.608, P=1.15 \times 10^{-58}\right)$, glutamate ionotropic receptor AMPA-type subunit $1(\mathrm{GRIA1}: \mathrm{r}=0.610, \mathrm{P}=2.39$ $\times 10^{-59}$ ), microfibrillar associated protein 4 (MFAP4: $\left.r=0.614, P=2.99 \times 10^{-60}\right)$, transcription factor $21($ TCF21: $r=$ $\left.0.627, \mathrm{P}=2.60 \times 10^{-63}\right)$, ADAM metallopeptidase with thrombospondin type 1 motif 8 (ADAMTS8: $r=0.624, P=$ $\left.1.66 \times 10^{-62}\right)$, FXYD domain-containing ion transport regulator $1\left(\mathrm{FXYD} 1: \mathrm{r}=0.654, \mathrm{P}=1.21 \times 10^{-70}\right)$, aspartoacylase (ASPA: $r=0.611, P=1.93 \times 10^{-59}$ ), four and a half LIM domains (FHL1: $r=0.625, P=5.50 \times 10^{-63}$ ), indolethylamine N-methyltransferase (INMT: $r=0.600, P=5.21 \times 10^{-57}$ ) and alcohol dehydrogenase 1B (class I), beta polypeptide (ADH1B: $r=0.644, \mathrm{P}=7.49 \times 10^{-68}$ ). Among these genes, INMT, FHL1, ADH1B, ASPA, FXYD1 and LINC00092 coexisted in an expressional module (Fig. 4b). In addition, TCGA data indicated that all of the above coding genes were downregulated in LUAD (Fig. 4c).

\section{Discussion}

Multiple studies have discovered a limited number of lincRNAs as diagnostic or prognostic biomarkers for several cancers, yet efforts are still needed to identify more lincRNAs with effects on cancer. Here, we demonstrated that LINC00092 suppresses growth of lung tumors and contributes to favorable survival in LUAD patients, which is controversial to its role in ovarian cancer according to a previously published study (Zhao, Ji et al., 2017). We found that LINC00092 is significantly downregulated in NSCLC tumorous tissues compared to that in normal lung tissues. Moreover, overexpression of LINC00092 inhibits LUAD cell proliferation and tumorigenic ability in vitro. 
Further studies are warranted to validate lincRNA functions in vivo and to explore possible molecular mechanisms.

LINC00092 can be activated by CAFs, and activated LINC00092 subsequently accelerates the effects of CAFs in tumorigenesis (Zhao, Ji et al., 2017). CAFs play a multifaceted role in cancer development (Cirri and Chiarugi, 2011), which has been documented to include the processes of proliferation (Li, Guan et al., 2016), progression (Hsu, Hung et al., 2016), metastasis (Ren, Zhou et al., 2016), chemoresistance (Choe, Shin et al., 2015), and radioresistance (Ji, Ji et al., 2015) in lung cancer cells. Similar to immune cells, CAFs initially inhibit malignant growth and early stages of tumor progression, while later, CAFs become activated and promote tumor development. Thus, CAF-mediated LINC00092 activation might play opposing roles in the early and advanced stages of cancers. The survival curve of LUAD patients in TCGA data further confirmed this hypothesis, as the favorable effect driven by LINC00092 tends to disappear or even become reversed as LUAD patients survive. This might be the reason why we achieved an inconsistent result in NSCLC to that published in ovarian cancer, where authors found that LINC00092 promotes ovarian cancer cell migration and invasion (Zhao, Ji et al., 2017), but our data revealed that LINC00092 does not affect the migratory or invasive ability of LUAD cells. In contrast, we found that LINC00092 inhibits tumor growth, which was not tested in ovarian cancer.

To illustrate how LINC00092 influences NSCLC development, we conducted WGCNA to assess possible coding genes related to LINC00092 and identified 11 coding genes. Among them, five genes and LINC00092 were coexpressed in a module including INMT, FHL1, ADH1B, ASPA, FXYD1. Kopantzev EP et al. reported that INMT and ADH1B were downregulated in lung cancer tissues compared to that in surrounding normal lung tissues but were more highly expressed in advanced stages of lung cancer than in earlier stages, further supporting the hypothesis that LINC00092 functions are reversed with cancer progression (Kopantzev, Monastyrskaya et al., 2008). FHL1 has also been reported to be downregulated and to play a growth inhibitory role in lung cancer (Niu, Liang et al., 2012). Additionally, these genes were all downregulated in NSCLC, as shown by TCGA data.

Although current studies and TCGA data showed that LINC00092 was consistently downregulated in both LUAD and LSCC samples, the lincRNA confers favorable survival only in LUAD patients but not in LSCC patients. These findings were further confirmed by TCGA data. Several studies have demonstrated that LUAD and LSCC display significant differences in their tumor molecular landscapes (Daraselia, Wang et al., 2012; Usui, Minami et al., 2013; Okamoto, Suzuki et al., 2014; Faruki, Mayhew et al., 2017). Several prognostic biomarkers such as Survivin (Sun, Jin et al., 2013), solute carrier family 16 member 1 (Stewart, Parapatics et al., 2015), and vascular invasion (Usui, Minami et al., 2013), have been proposed for LUAD but not for LSCC. However, few studies have reported that lincRNAs confer differences in survival between LUAD and LSCC patients, although several studies have investigated some lincRNAs in LUAD or LSCC alone. To the best of our knowledge, our data suggest that a lincRNA is significantly associated with LUAD prognosis but not with LSCC prognosis. All these biomarkers further can increase the accuracy for individualized targeted therapy.

In conclusion, our data revealed that LINC00092 is downregulated in NSCLC and is correlated with the expression of several coding genes. High expression of LINC00092 contributes to favorable survival in LUAD patients but not in LSCC patients. Upregulation of LINC00092 can repress tumor growth in vitro. These results indicate that LINC00092 is a possible predictive biomarker for LUAD. Targeted molecular therapy directed at LINC00092 upregulation may be a valuable strategy to fight LUAD. 


\section{Conclusions}

LINC00092 is an indicator of favorable LUAD prognosis. Targeted molecular therapy directed at LINC00092 upregulation may be a valuable strategy to fight LUAD.

\section{Abbreviations}

\section{CAFs}

cancer-associated fibroblasts

LINC00092

Long intergenic noncoding RNA 00092

LUAD

low expression in lung adenocarcinoma

LSCC

lung squamous cell carcinoma

LincRNAs

Long intergenic noncoding RNAs

NSCLC

non-small cell lung cancer

TCGA

The Cancer Genome Atlas

\section{Declarations}

Competing interests

All authors read and approved the manuscript. The authors declare that they have no competing interests as defined by Nature Research or other interests that might be perceived to influence the results and/or discussion reported in this paper.

Funding

This work was supported by The Natural Science Foundation of Guangdong Province (2017A030313860). The funders had no role in study design, data collection and analysis, decision to publish, or manuscript preparation. Authors' contributions $\mathrm{MJ}$ and $\mathrm{KW}$ collected and checked the data, planned the statistical analysis, and analyzed the data. ZWZ, YL, HH, and SW monitored data collection, conducted the statistical analysis and revised the paper. QC, MC and JX conducted lung tissue collection from patients and individuals. ZXZ initiated and designed the study, monitored the data analysis, and drafted and revised the manuscript.

Acknowledgement

We thank Lunlun Zhu for assisting us with the tissue sample collection.

\section{References}

1.

Siegel RL, Miller KD, Jemal A. Cancer statistics, 2016. CA Cancer J Clin. 2016;66:7-30.

2. 
Chen W, Zheng R, Baade PD, et al. Cancer statistics in China, 2015. CA Cancer J Clin. 2016;66:115-32.

3.

Siegel RL, Miller KD, Jemal A. Cancer statistics, 2015. CA Cancer J Clin. 2015;65:5-29.

4.

Chen G, Kronenberger P, Teugels E, Umelo IA, De Greve J. Targeting the epidermal growth factor receptor in nonsmall cell lung cancer cells: the effect of combining RNA interference with tyrosine kinase inhibitors or cetuximab. BMC Med. 2012;10:28.

5.

Chee J, Robinson BW, Holt RA, Creaney J. Immunotherapy for Lung Malignancies: From Gene Sequencing to Novel Therapies. Chest. 2017;151:891-7.

6.

Ma C, Wu G, Zhu Q, et al.: Long intergenic noncoding RNA 00673 promotes non-small-cell lung cancer metastasis by binding with EZH2 and causing epigenetic silencing of HOXA5. Oncotarget, 2017.

2017

Ma C, Wu G, Zhu Q, et al.: Long intergenic noncoding RNA 00673 promotes non-small-cell lung cancer metastasis by binding with EZH2 and causing epigenetic silencing of HOXA5. Oncotarget, 2017.

7.

Sun CC, Li SJ, Li G, Hua RX, Zhou XH, Li DJ. Long Intergenic Noncoding RNA 00511 Acts as an Oncogene in Nonsmall-cell Lung Cancer by Binding to EZH2 and Suppressing p57. Mol Ther Nucleic Acids. 2016;5:e385.

8.

Li W, Li H, Zhang L, et al. Long non-coding RNA LINC00672 contributes to p53 protein-mediated gene suppression and promotes endometrial cancer chemosensitivity. J Biol Chem. 2017;292:5801-13.

9.

Panzitt K, Tschernatsch MM, Guelly C, et al. Characterization of HULC, a novel gene with striking up-regulation in hepatocellular carcinoma, as noncoding RNA. Gastroenterology. 2007;132:330-42.

10.

Russell MR, Penikis A, Oldridge DA, et al. CASC15-S Is a Tumor Suppressor IncRNA at the 6p22 Neuroblastoma Susceptibility Locus. Cancer Res. 2015;75:3155-66.

11.

Zhao L, Ji G, Le X, et al. Long Noncoding RNA LINC00092 Acts in Cancer-Associated Fibroblasts to Drive Glycolysis and Progression of Ovarian Cancer. Cancer Res. 2017;77:1369-82.

12.

Micke P, Ostman A. Tumour-stroma interaction: cancer-associated fibroblasts as novel targets in anti-cancer therapy? Lung Cancer. 2004;45(Suppl 2):163-75.

13.

Cirri P, Chiarugi P. Cancer associated fibroblasts: the dark side of the coin. Am J Cancer Res. 2011;1:482-97. 14.

Li J, Guan J, Long X, Wang Y, Xiang X. mir-1-mediated paracrine effect of cancer-associated fibroblasts on lung cancer cell proliferation and chemoresistance. Oncol Rep. 2016;35:3523-31.

15.

Hsu YL, Hung JY, Chiang SY, et al. Lung cancer-derived galectin-1 contributes to cancer associated fibroblastmediated cancer progression and immune suppression through TD02/kynurenine axis. Oncotarget. 2016;7:27584-98. 
16.

Ren Y, Zhou X, Liu X, et al. Reprogramming carcinoma associated fibroblasts by AC1MMYR2 impedes tumor metastasis and improves chemotherapy efficacy. Cancer Lett. 2016;374:96-106.

17.

Choe C, Shin YS, Kim C, et al. Crosstalk with cancer-associated fibroblasts induces resistance of non-small cell lung cancer cells to epidermal growth factor receptor tyrosine kinase inhibition. Onco Targets Ther. 2015;8:366578.

18.

Ji X, Ji J, Shan F, Zhang Y, Chen Y, Lu X. Cancer-associated fibroblasts from NSCLC promote the radioresistance in lung cancer cell lines. Int J Clin Exp Med. 2015;8:7002-8.

19.

Kopantzev EP, Monastyrskaya GS, Vinogradova TV, et al. Differences in gene expression levels between early and later stages of human lung development are opposite to those between normal lung tissue and non-small lung cell carcinoma. Lung Cancer. 2008;62:23-34.

20.

Niu C, Liang C, Guo J, et al. Downregulation and growth inhibitory role of FHL1 in lung cancer. Int J Cancer. 2012;130:2549-56.

21.

Faruki H, Mayhew GM, Serody JS, Hayes DN, Perou CM, Lai-Goldman M. Lung Adenocarcinoma and Squamous Cell Carcinoma Gene Expression Subtypes Demonstrate Significant Differences in Tumor Immune Landscape. J Thorac Oncol, 2017.

22.

Okamoto T, Suzuki Y, Fujishita T, et al. The prognostic impact of the amount of tobacco smoking in non-small cell lung cancer-differences between adenocarcinoma and squamous cell carcinoma. Lung Cancer. 2014;85:125-30. 23.

Usui S, Minami Y, Shiozawa T, et al. Differences in the prognostic implications of vascular invasion between lung adenocarcinoma and squamous cell carcinoma. Lung Cancer. 2013;82:407-12.

24.

Daraselia N, Wang Y, Budoff A, et al. Molecular signature and pathway analysis of human primary squamous and adenocarcinoma lung cancers. Am J Cancer Res. 2012;2:93-103.

25.

Sun PL, Jin Y, Kim H, et al. Survivin expression is an independent poor prognostic marker in lung adenocarcinoma but not in squamous cell carcinoma. Virchows Arch. 2013;463:427-36.

26.

Stewart PA, Parapatics K, Welsh EA, et al. A Pilot Proteogenomic Study with Data Integration Identifies MCT1 and GLUT1 as Prognostic Markers in Lung Adenocarcinoma. PLoS One. 2015;10:e0142162.

27.

Yang L, Yang X, Ji W, et al. Effects of a functional variant c.353T > C in snai1 on risk of two contextual diseases. Chronic obstructive pulmonary disease and lung cancer. Am J Respir Crit Care Med. 2014;189:139-48.

28.

Langfelder P, Horvath S. WGCNA: an R package for weighted correlation network analysis. BMC Bioinformatics. 2008;9:559.

29. 
Shannon P, Markiel A, Ozier O, et al. Cytoscape: a software environment for integrated models of biomolecular interaction networks. Genome Res. 2003;13:2498-504.

\section{Figures}

A

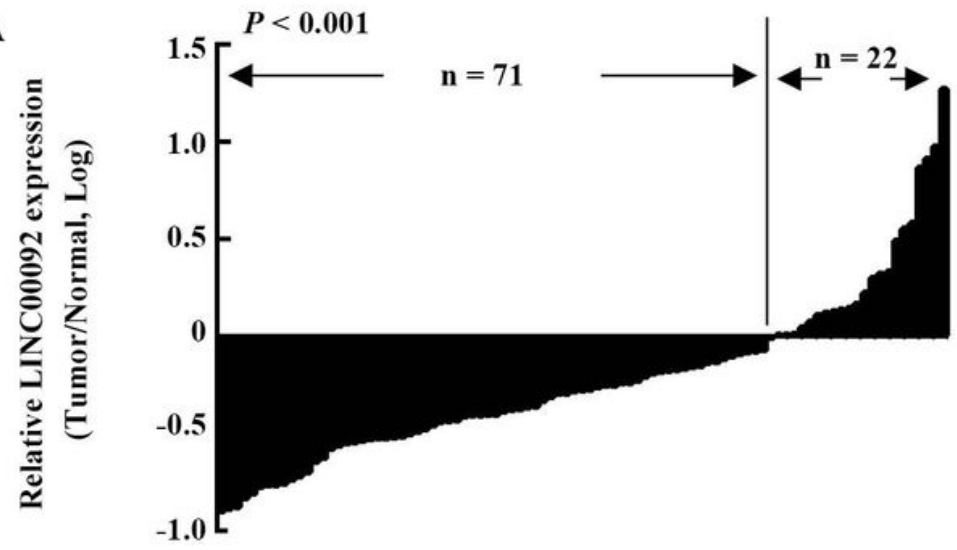

B

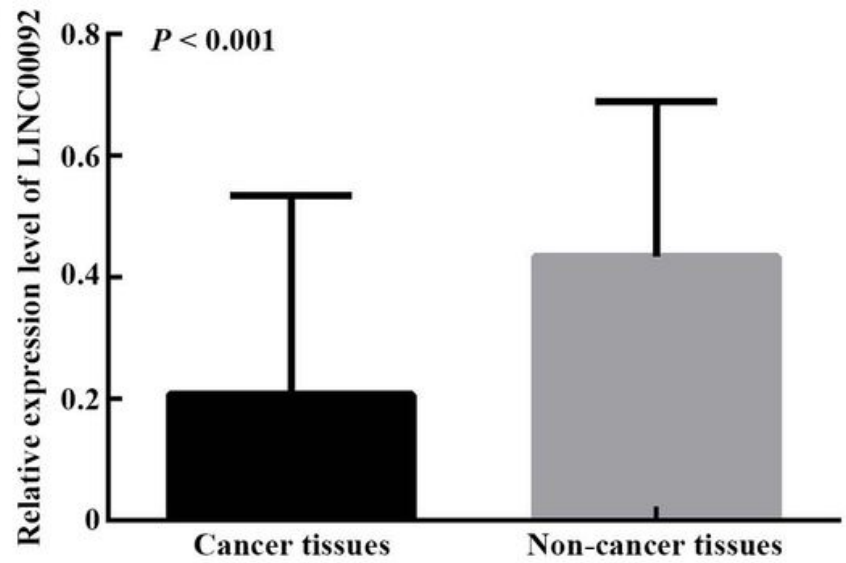

C

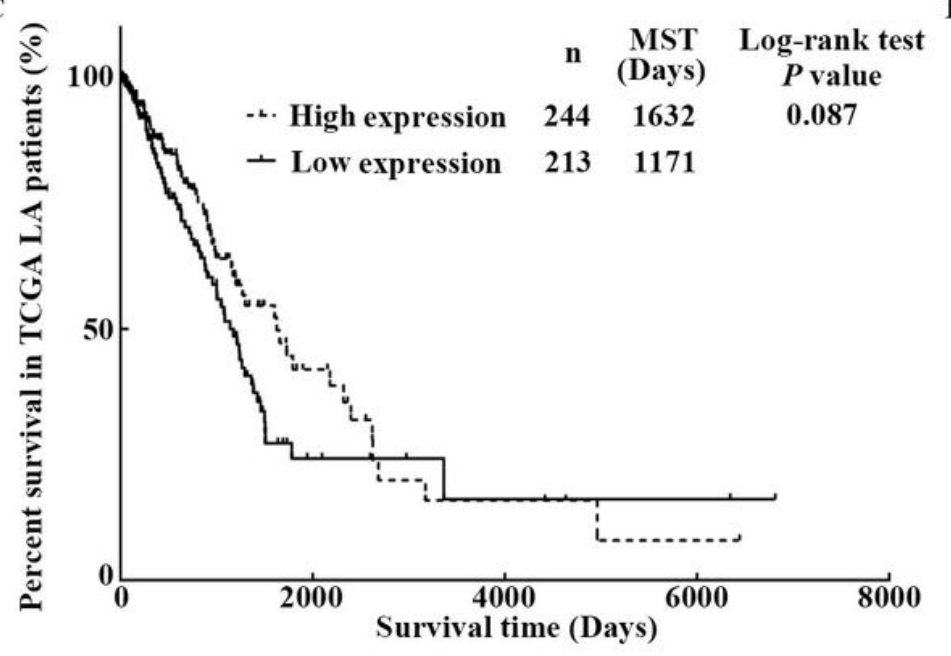

D

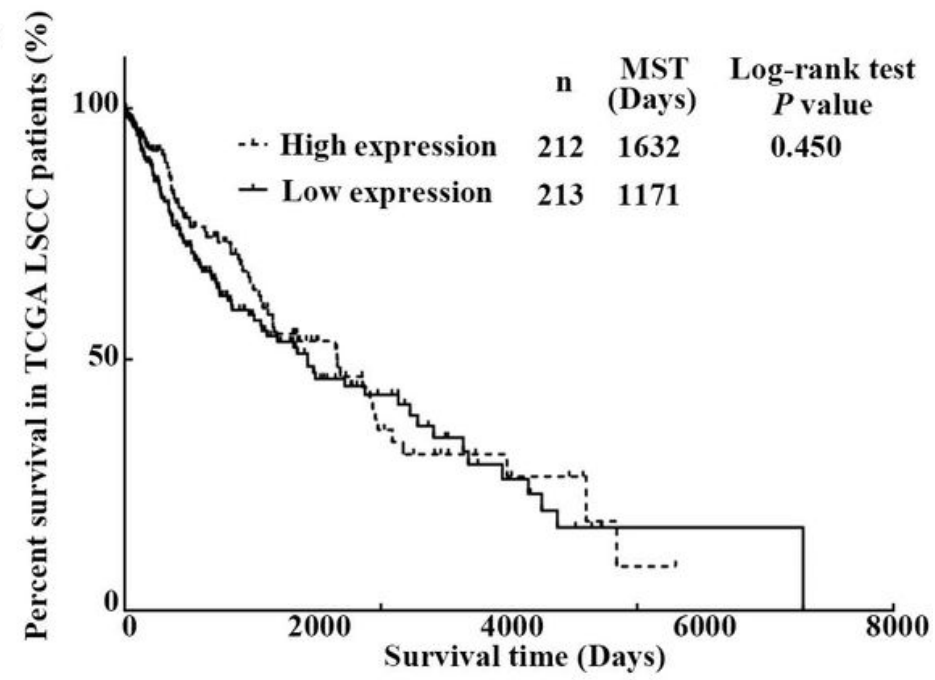

\section{Figure 1}

Downregulation of LINC00092 in NSCLC. a-b. Expression pattern of LINC00092 in NSCLC tissues. LINC00092 was significantly downregulated in NSCLC tissues compared to that in adjacent normal lung tissues. Results are shown as tumor-normal pairs. P, calculated using paired t tests (a) and presented as the mean \pm SD. P, calculated using Student's t tests (b). The expression level for each tissue was from three replicates. c-d. Effect of LINC00092 expression on the survival of LUAD (c) and LSCC (d) patients based on TCGA data. LUAD patients with low expression of LINC00092 exhibited significantly poorer survival than patients with high expression of LINC00092. $\mathrm{P}$, calculated using the log-rank test. 

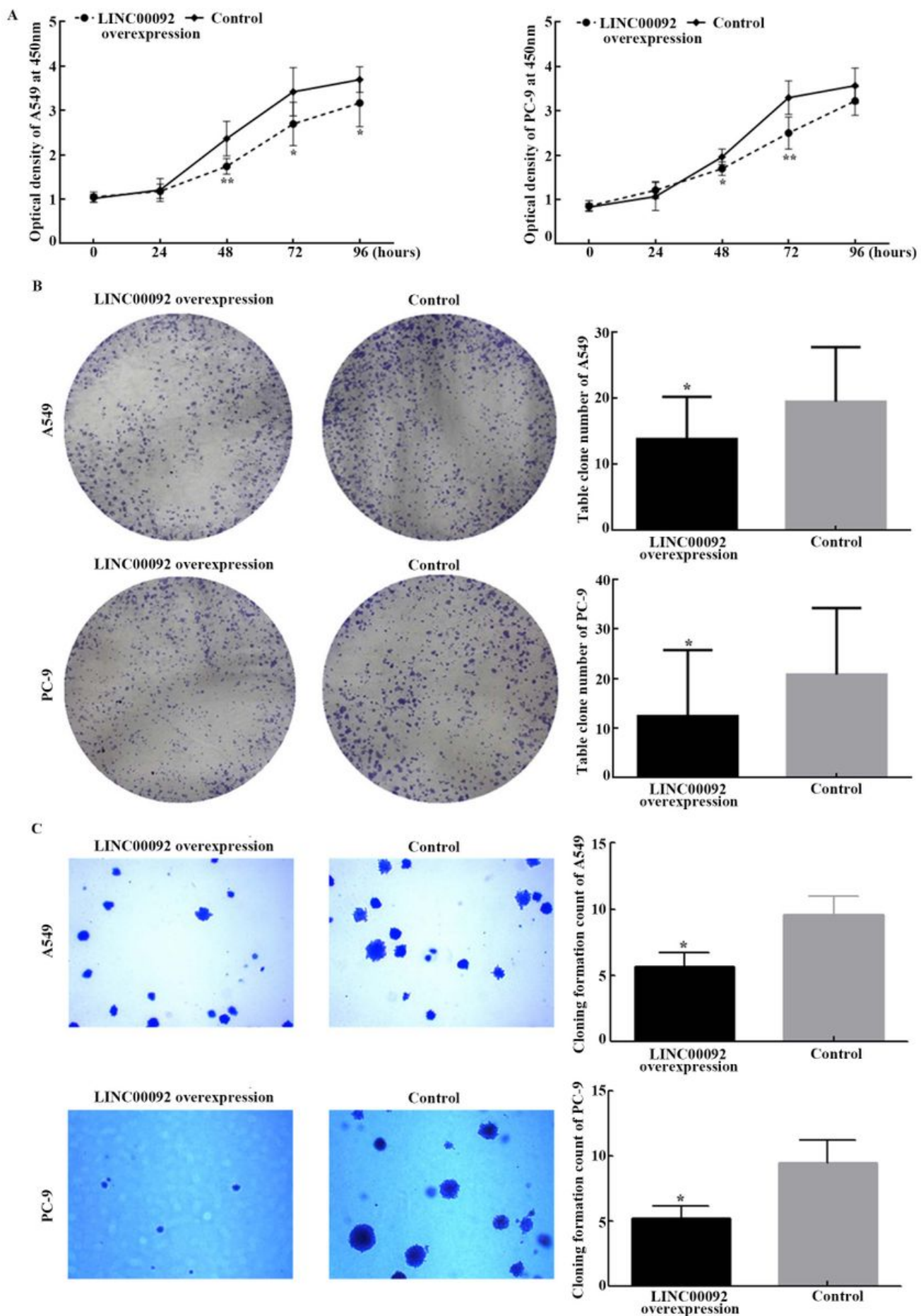

Figure 2

Effects of LINC00092 on malignant phenotypes in LUAD. a. Effect of LINC00092 on cell proliferation. Both A549 and PC9 cell lines that overexpressed LINC00092 exhibited a significantly lower proliferation rate than the corresponding control cell lines. b-c, Effect of LINC00092 on colony formation. Both plate colony (b) and soft agar assays (c) showed that the upregulation of LINC00092 impaired the tumorigenic abilities of both A549 and PC9 cell lines. Results are shown as the mean \pm SD. P, calculated using Student's t tests. 
A
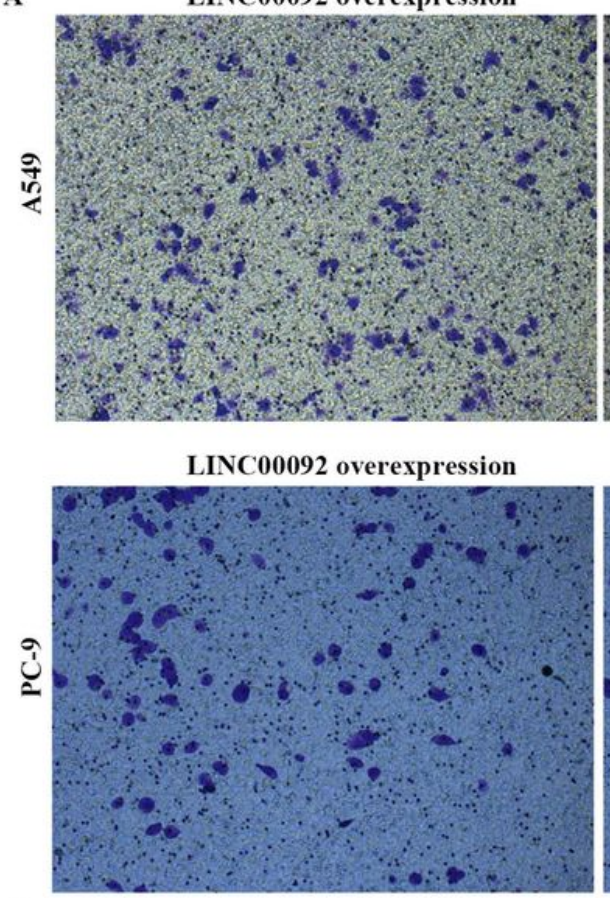

B

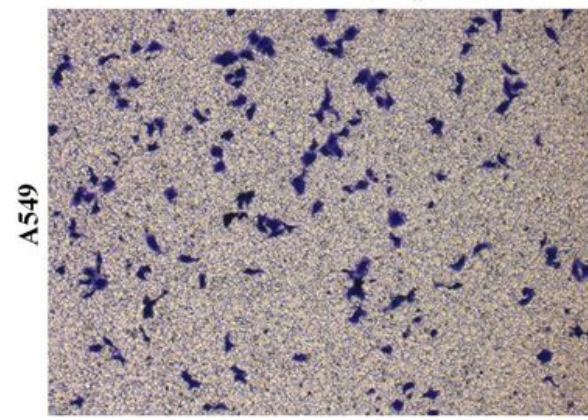

LINC00092 overexpression

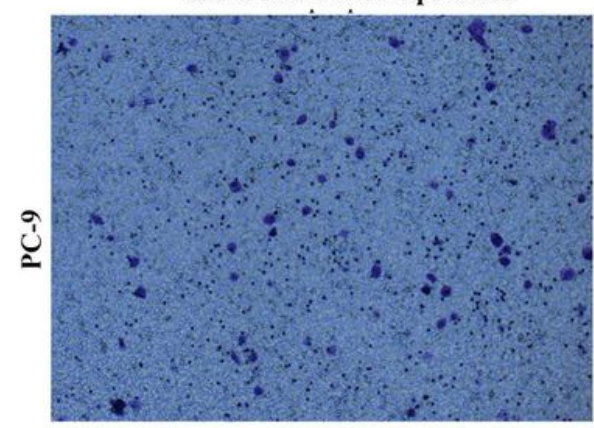

Control

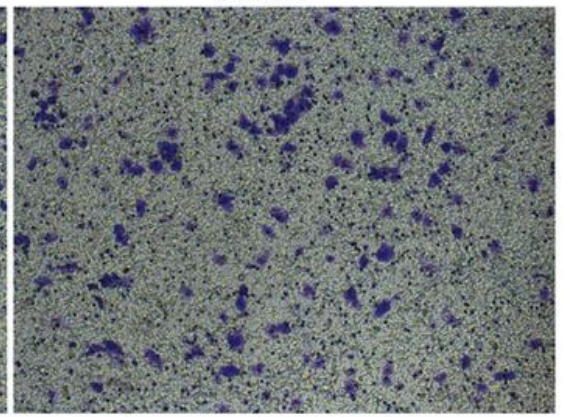

Control

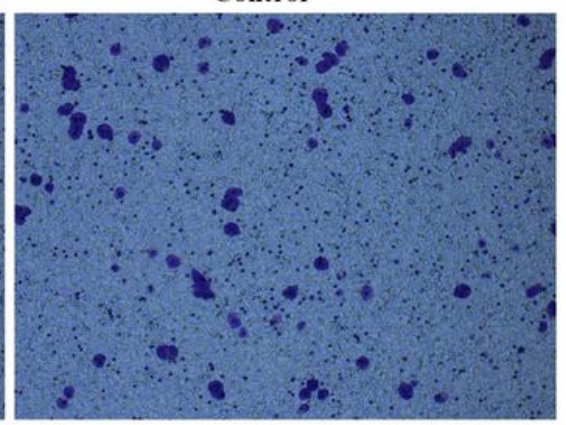

Control

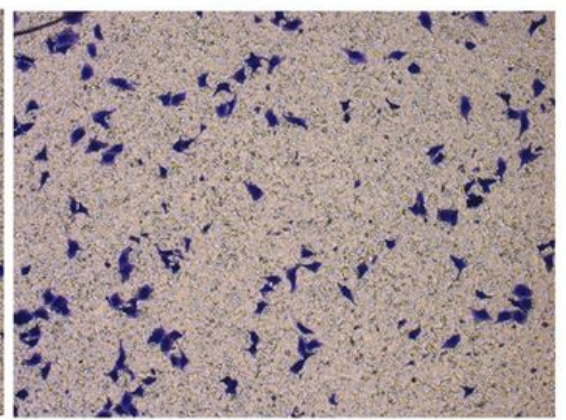

Control

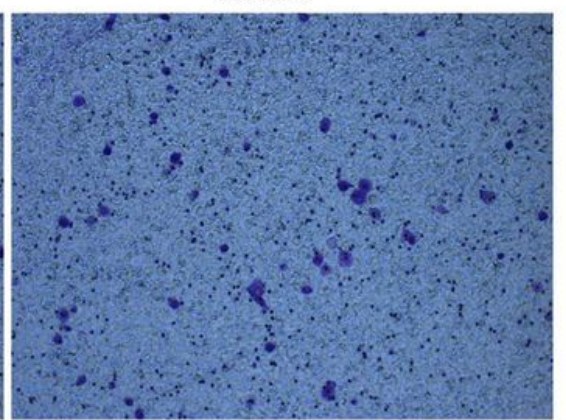

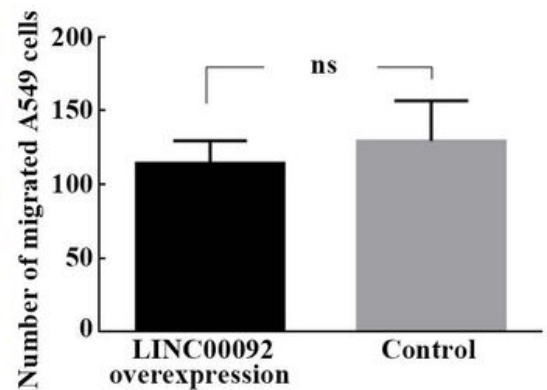
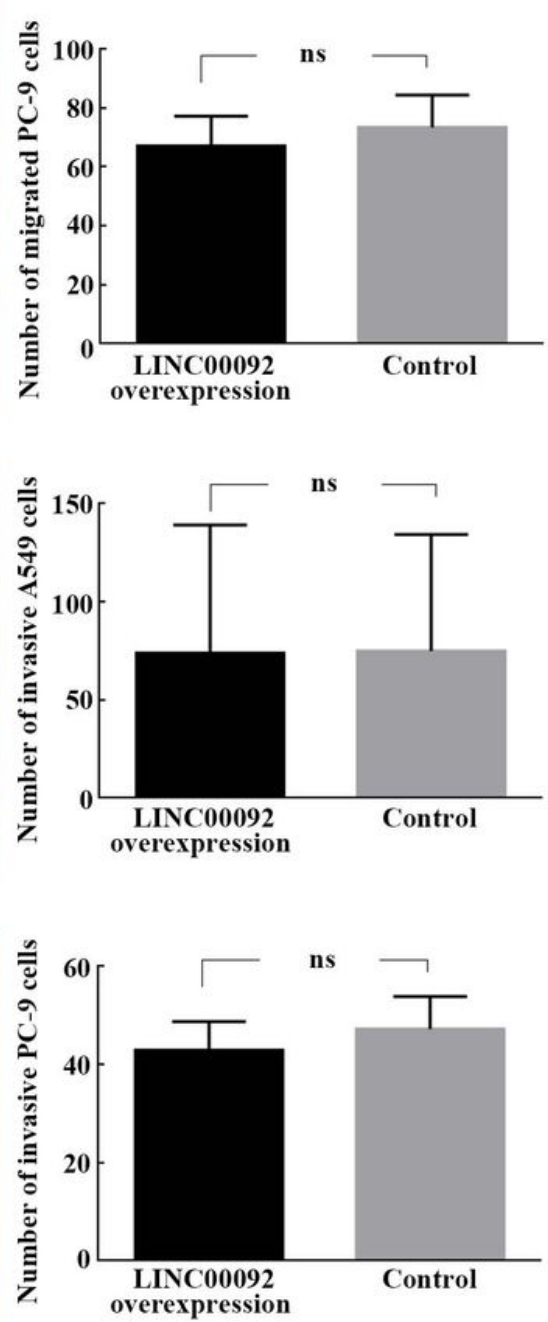

Figure 3

Effects of LINC00092 on cell migration and invasion. a. Cell migration. Upregulation of LINC00092 did not significantly affect the migratory ability of either A549 or PC9 cells. b. Cell invasion. Upregulation of LINC00092 did not significantly affect the invasive ability of either A549 or PC9 cells. Results are shown as the mean \pm SD. P, calculated using Student's t tests. 
A

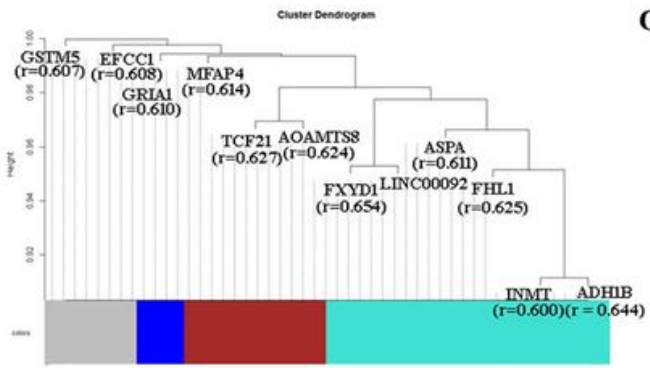

B

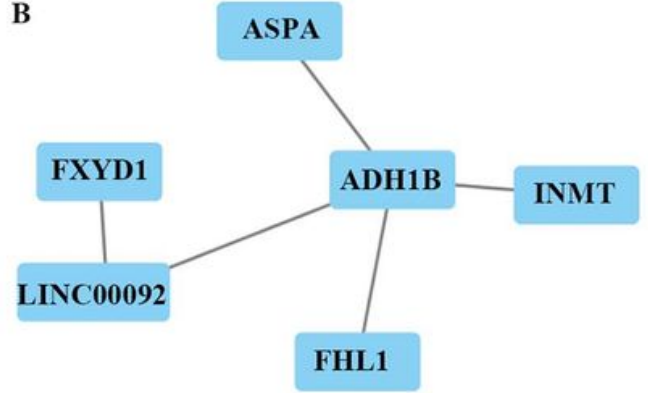

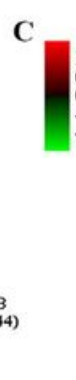

Non-cancer

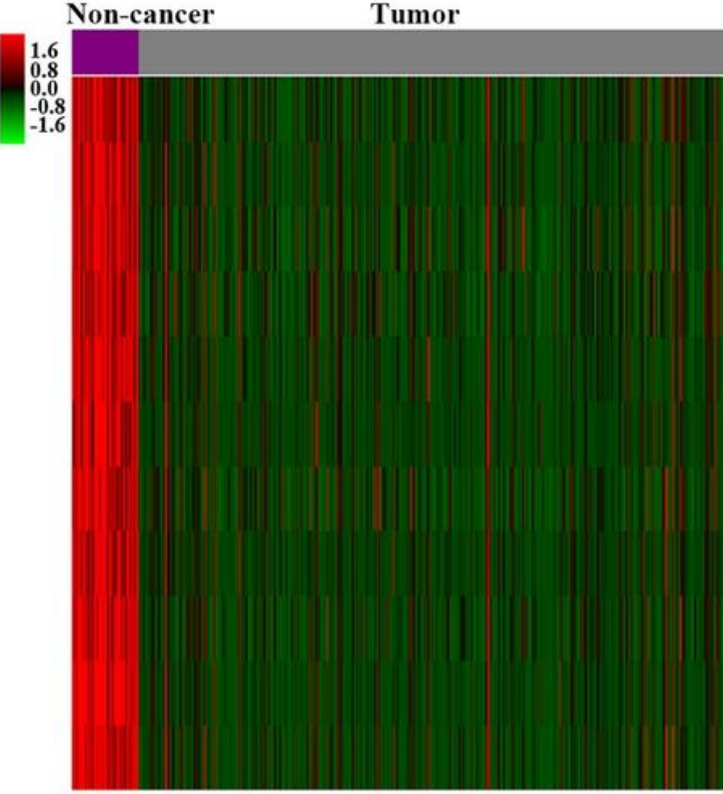

Gene $\quad \log 2$

ID Foldchange

FDR adjusted

GSTM5 -2.409

TCF21 -3.079

MFAP4 -2.595

EFCC1 -2.540

AOAMTS8 -3.457

GRIA1 $-\mathbf{- 3 . 6 9 5}$

ASPA $\quad \mathbf{- 2 . 6 5 8}$

INMT $\quad \mathbf{- 3 . 4 7 7}$

FXYD1 -2.896

FHL1 $\quad-3.407$

ADH1B $\quad \mathbf{- 3 . 2 3 7}$
$P$ value

$2.48 \times 10^{-56}$

$6.81 \times 10^{-55}$

$2.76 \times 10-46$

$1.10 \times 10-62$

$6.17 \times 10^{-52}$

$1.20 \times 10^{-40}$

$3.10 \times 10^{-51}$

$5.57 \times 10-64$

$1.52 \times 10-66$

$8.88 \times 10^{-83}$

$1.04 \times 10^{-33}$

\section{Figure 4}

Gene coexpression network with regard to LINC00092. a. WGCNA showed a series of coding genes displaying significant correlations with LINC00092 expression b. WGCNA showed the expressional module of LINC00092. c. TCGA data showed that all these correlated coding genes were downregulated in LUAD. 